\title{
Antenatal and Postnatal Diagnosis of Coxsackie B4 Infection: Case Series
}

\author{
Jennifer C. Hunt, M.D., F.R.C.S.C. ${ }^{1}$ Carol Schneider, M.D., F.R.C.S.C. ${ }^{1}$ \\ Savas Menticoglou, M.D., F.R.C.S.C. ${ }^{1}$ Jayantha Herath, M.D., F.R.C.P.C. ${ }^{2}$ \\ Marc R. Del Bigio, M.D., Ph.D., F.R.C.P.C. ${ }^{2}$
}

${ }^{1}$ Department of Obstetrics and Gynaecology

2 Department of Pathology, University of Manitoba, Winnipeg,

Address for correspondence and reprint requests Jennifer C. Hunt, Manitoba, Canada

M.D., F.R.C.S.C., Department of Obstetrics and Gynaecology,

Women's Hospital, Room WS125, 735 Notre Dame Avenue, Winnipeg,

Am J Perinatol Rep 2012;2:1-6.

Manitoba, Canada R3E 0L8 (e-mail: drjenhunt@gmail.com).

\begin{abstract}
Keywords

- coxsackie B virus

- hydrops

- myocarditis

- cerebritis

- ventriculitis

Enteroviruses are a common cause of neonatal infection. In particular, Coxsackie B viruses are often associated with severe, fatal disease. The antenatal diagnosis of Coxsackie B viral infections is uncommon. We present a unique case of Coxsackie B4 virus ventriculitis and myocarditis causing fetal hydrops at 22 weeks gestation. Transmission was inferred by viral isolation from the amniotic fluid and by placental pathology. We also describe two additional cases of fatal neonatal Coxsackie B4 infection complicated by myocarditis and encephalitis with cerebral necrosis in a 4-day-old female and by myocarditis, spinal leptomeningitis, and hepatitis in a 4-day-old male. Transplacental acquisition of infection carries a poor prognosis. We propose that Coxsackie B virus should be considered in the investigation of nonimmune hydrops, particularly in the presence of cardiac dysfunction.
\end{abstract}

Enteroviruses, which include Coxsackie viruses and echoviruses, are a common cause of infection in neonates and infants less than 1 year old. ${ }^{1-3}$ The spectrum of disease can range from a nonspecific febrile illness to a severe, lifethreatening multiorgan infection. ${ }^{3,4}$ The transmission of enterovirus from mother to neonate is usually through contact with infected maternal secretions at the time of vaginal delivery. $^{2-6}$

Transplacental transmission of enteroviruses has also been described and is typically associated with more severe, and often fatal, disease. ${ }^{2,6,7}$ In these cases, onset of illness is usually within the first few days of life., ${ }^{2,8}$ Most mothers will have experienced symptoms in the 1 to 2 weeks before delivery $^{2,6,9}$; however, the neonate may acquire the infection in the absence of maternal symptoms. ${ }^{10}$ Maternal clinical syndromes include a nonspecific febrile illness, upper respiratory tract illness, pleurodynia, meningitis, and abdominal pain. $^{2,7}$

The group B Coxsackie viruses (particularly types B1 to B4) are most frequently associated with severe and fatal neonatal disease. ${ }^{2,6}$ The most common neonatal manifestations include myocarditis, 2,6,10-12 meningoencephalitis, 2,6,7,9,10,13 hepatitis, $2,6,8,10,11$ disseminated intravascular coagulopathy, $2,6,8,11$ and sepsis-like syndromes. ${ }^{2,11}$ Less common presentations include pneumonia, gastrointestinal symptoms, pancreatitis, cutaneous exanthems, and seizures. ${ }^{3,5,7}$ Mortality rates are highest with myocarditis and hepatitis in association with disseminated intravascular coagulopathy., 2,6 Congenital infection has also been linked to childhood type I diabetes, ${ }^{14,15}$ neurodevelopmental delays, ${ }^{16}$ structural cardiac anomalies, ${ }^{17}$ and severe anatomic defects of the central nervous system (CNS) such as hydranencephaly and hydrocephalus. ${ }^{18}$ In a review by Kamei et al, all previously reported fatal cases of Coxsackie B encephalitis and myocarditis had occurred within hours after birth to 14 days of age, with more than half of cases attributable to Coxsackie B4 virus. ${ }^{19}$

We report a case of severe fetal hydrops at 22 weeks gestation secondary to myocarditis. Coxsackie B4 virus was isolated from the amniotic fluid. This prompted us to conduct a retrospective review at our center of perinatal deaths received

July 28, 2011

accepted after revision

August 27, 2011

published online

November 25, 2011
Copyright (C) 2012 by Thieme Medical Publishers, Inc., 333 Seventh Avenue, New York, NY 10001, USA. Tel: +1(212) 584-4662.
DOI http://dx.doi.org/

10.1055/s-0031-1296027.

ISSN 2157-6998. 
associated with Coxsackie viral infections presumably acquired by vertical transmission.

\section{Methods}

Pediatric autopsy records at the Health Sciences Centre were searched retrospectively from 1980 to 2010 using a whole text search tool (ISYS Search Software, Denver, CO) to find the word "Coxsackie." The research was conducted with approval of the Research Ethics Board of the University of Manitoba (protocol H2011:051). All pediatric and $>95 \%$ of fetal autopsies in the province of Manitoba (population 1.1 million; 120 autopsies per year) are conducted at the center. Reports were reviewed to verify that Coxsackie infection had been proven and then the available hospital charts and microscopy slides were reviewed. One additional earlier case was identified.

For pathological review, in all three cases the hematoxylin and eosin stained sections of all organs were examined. We stained selected slides from the brain and heart with Perls Prussian blue method for hemosiderin, chloracetate esterase (Leder) method for neutrophils, and von Kossa method for calcium. Immunohistochemical stains were performed to demonstrate glial fibrillary acidic protein (a marker for reactive astrocytes), human leukocyte antigen (a marker for reactive microglia and macrophages), CD3, CD4, CD8 (markers for T lymphocytes and subpopulations), cytomegalovirus (CMV), and Herpes virus proteins. Small samples of abnormal periventricular tissue from the fetal brain were additionally fixed in glutaraldehyde and osmium tetroxide then embedded in plastic resin for electron microscopic examination.

\section{Case 1}

A 24-year-old G3P1SA1 was referred to our center at 22 weeks gestation. She was healthy, apart from a history of bilateral congenital sensorineural hearing loss. The pregnancy had been uneventful until $\sim 17$ to 18 weeks when the patient experienced a low-grade fever. The patient recovered; however, her husband required admission to a community hospital with a diagnosis of viral meningitis. At 20 weeks gestation, intermittent fetal tachycardia was noted at a routine prenatal visit. A fetal ultrasound examination performed 21 weeks gestation showed significant fetal body wall edema involving the scalp, neck, and abdomen. There was mild abdominal ascites. Fetal tachycardia was confirmed. Placentomegaly was also seen.

At 22 weeks gestation, the patient was referred to our center with a preliminary diagnosis of nonimmune fetal hydrops. Ultrasonography again confirmed the presence of fetal ascites, body wall edema, and pleural effusions (-Fig. 1A). No structural cardiac anomalies were appreciated. The gut, liver, and heart were echogenic and bilateral lateral ventriculomegaly was present (-Figs. 1B and 1C). A large, hydropic placenta was also identified ( - Fig. 1D). Intermittent fetal tachycardia was documented (maximum 260 to 280 bpm). The observed fetal hydrops was attributed to supraventricular tachycardia (SVT) and the mother was started on sotalol $40 \mathrm{mg}$ twice daily.
In the following days, the fetal tachycardia resolved completely; however, the hydrops and cardiac dysfunction worsened. Fetal movements were also reduced. The antiarrhythmic therapy was discontinued because the clinical findings no longer seemed compatible with the initial diagnosis of SVT. Maternal serology for parvovirus B19, toxoplasmosis, CMV, and syphilis were negative. She was immune to both varicella and rubella. The fetal karyotype was confirmed to be that of a normal male (46 XY). Amniotic fluid testing for toxoplasmosis, CMV, and parvovirus B19 was negative. Viral studies for enterovirus were positive. Subsequent polymerase chain reaction (PCR) identified Coxsackie B4 virus from the amniotic fluid sample.

The fetal condition further deteriorated between 23 and 24 weeks gestation with worsening hydrops, decreasing amniotic fluid volume, and a reduction in fetal movements. On the final visit, the maternal blood pressure was slightly elevated (140/90). Screening blood work for pre-eclampsia was normal with the exception of an elevated uric acid level (332 $\mu \mathrm{mol} / \mathrm{L}$ ). With a concern for the development of maternal mirror syndrome, combined with a poor fetal prognosis, the pregnancy was electively terminated by induction of labor with vaginal misoprostol. A stillborn male infant was delivered (body weight $690 \mathrm{~g}$ ). The scalp, neck, abdomen, and limbs were edematous but no dysmorphic features were noted.

The fetus and placenta were submitted for pathologic examination. Extensive dystrophic calcifications were found on the inferior surface of the liver and in the right atrium of the heart. Lymphocytic infiltrates were noted within the myocardium, consistent with a viral myocarditis (-Fig. 2C). Examination of the brain showed enlargement of the lateral ventricles with severe multifocal ventriculitis characterized by loss of ependymal cells, $\mathrm{T}$ lymphocyte infiltrate, microglial activation, small foci of hemorrhage, dystrophic calcification, and clusters of hemosiderin-containing macrophages (-Figs. 2A and 2B). Electron microscopy failed to identify viral inclusions. In addition, immunostaining for T lymphocytes showed a mild leptomeningitis. The placental disc was large (>90th percentile) and the chorionic villi showed evidence of a chronic inflammatory infiltrate with dystrophic calcifications. No viral inclusions were visualized in the placental tissue. Cultures of lung, stool, and placental tissue were negative for virus and bacteria.

\section{Case 2}

A 4-day-old female infant presented with fever, jaundice, and lethargy. The baby was born at 37 weeks gestation to a 29year-old G3P1 mother via caesarean section for failure to progress in labor and suspected chorioamnionitis (maternal fever and fetal tachycardia). The Apgar scores were 8 and 8 at 1 and 5 minutes, respectively. The birth weight was $2771 \mathrm{~g}$. One week before birth, the infant's sibling had been unwell with a viral illness.

The infant was initially treated with antibiotics for sepsis; however, blood and cerebrospinal fluid (CSF) cultures were negative for bacteria. At 7 days of age, a chest X-ray revealed cardiomegaly. An echocardiogram showed poor myocardial 
A
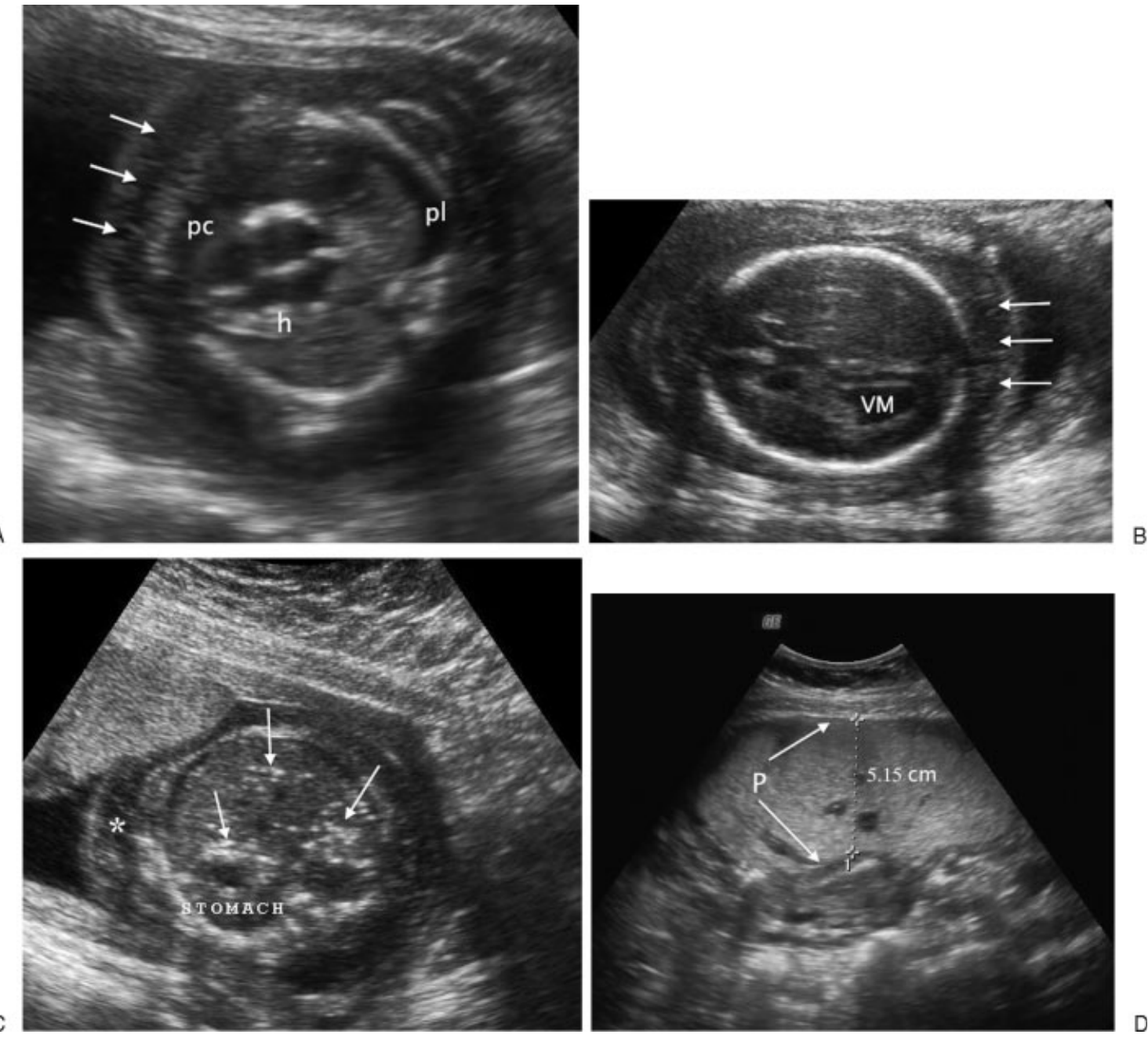

Figure 1 2D gray scale ultrasound images at 22 weeks gestation of the fetus described in Case 1. (A) Transverse axial view of the fetal chest with skin edema (arrows); cardiac hypertrophy (h); pericardial (pc); and pleural (pl) effusions. (B) Transverse axial view of the fetal brain showing ventriculomegaly (vm) and scalp edema (arrows). (C) Transverse axial view of the fetal abdomen showing body wall edema $\left({ }^{*}\right)$ and intra-abdominal calcifications (arrows). (D) The ultrasound findings also revealed associated placentomegaly (p).

function, dilatation of the atria, moderate mitral and tricuspid regurgitation, and dilatation of the ventricles. An incidental finding of a small muscular ventriculoseptal defect was not felt to account for the degree of cardiac dysfunction.

Subsequent stool and throat cultures were positive for Coxsackie B4 virus at 16 days of age leading to a diagnosis of viral myocarditis. The infant's clinical course was complicated by congestive heart failure, coagulase negative Staphylococcus sepsis, left lung collapse, thrombocytopenia with pulmonary and gastrointestinal bleeding, renal failure, and metabolic disturbances. The neonate also developed seizures and tense fontanelles, leading to a clinical suspicion of encephalitis. Subsequent cerebral ultrasonography revealed bilateral grade IV intraventricular hemorrhages, ventriculomegaly, and a porencephalic cyst.

By 28 days of age, severe cardiomyopathy persisted despite aggressive medical management. Support was withdrawn and the infant died shortly thereafter. Autopsy revealed an enlarged heart ( $50 \mathrm{~g}$, normal $21 \mathrm{~g}$ ) and a large amount of peritoneal ascites. The lungs were congested with profuse intra-alveolar hemosiderin-containing macrophages. The left ventricle of the heart had severe myocarditis with extensive dystrophic calcifications. Viral inclusions were not present. The liver had widespread pericentral hepatocyte degeneration and fibrosis. The kidneys were also congested with focal areas of calcification, fibrosis, inflammation, and sclerosis. Viral cultures of the lung, heart, liver, and kidney were negative.

The brain was mildly edematous ( $482 \mathrm{~g}$, normal $433 \mathrm{~g}$ ). There was extensive hemorrhage and necrosis involving the cerebral and cerebellar white matter. In addition, areas of subarachnoid and intraventricular hemorrhage were evident. Deposits of calcium and hemosiderin were seen adjacent to the areas of hemorrhage. However, there was no lymphocytic infiltrate in the brain or meninges suggesting that the brain changes were secondary. The cause of death was attributed to 


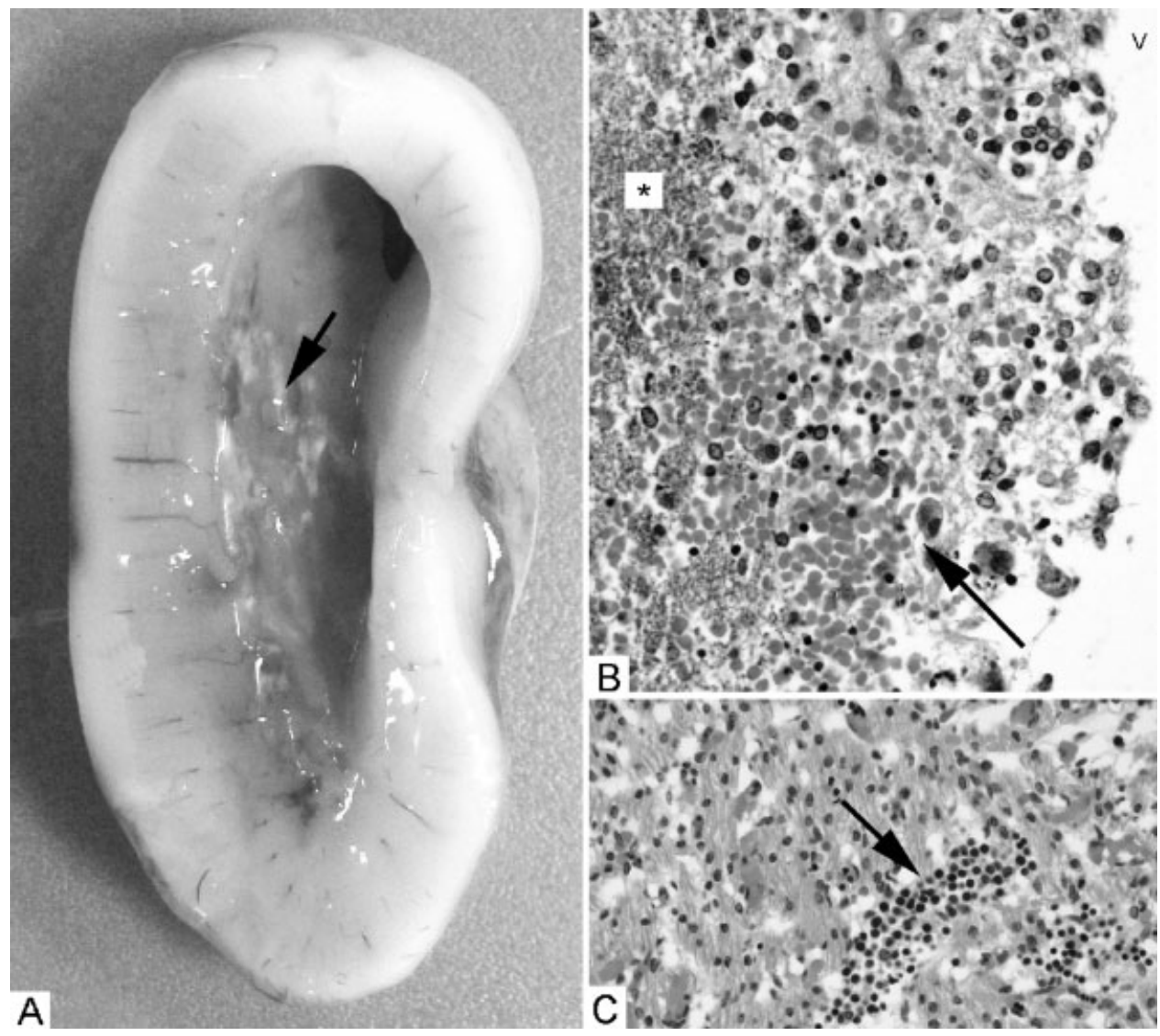

Figure 2 (A) Coronal slice through left parietal lobe of brain showing enlarged lateral ventricle with mottled discoloration of wall including focal pale areas of calcification (arrow). (B) Photomicrograph showing ventricular (V) surface of brain with absence of ependymal lining, blood with macrophages and lymphocytes (arrow), and calcification $\left({ }^{*}\right)$. (C) Photomicrograph showing myocardium of left ventricle focally infiltrated by lymphocytes (arrow). (B and C both hematoxylin and eosin stain, $400 \times$ magnification.)

neonatal myocarditis due to Coxsackie B4 virus, possibly acquired in utero given the intrapartum maternal symptoms and the history of illness in the family.

\section{Case 3}

A 4-day-old male infant presented with lethargy and poor feeding. The baby was born uneventfully by vaginal delivery at term, with a birth weight of $3572 \mathrm{~g}$. His mother had been well until 2 days before delivery when she developed a flulike illness and a fever of $39.4^{\circ} \mathrm{C}$. Progressive jaundice appeared by 6 days of age with marked hepatomegaly and indirect hyperbilirubinemia. Upon admission to hospital, the infant was febrile $\left(38.9^{\circ} \mathrm{C}\right)$. Meningitis was suspected on the basis of lymphocytic pleocytosis (100 cell $/ \mathrm{mm}^{3}$; 95\% lymphocytes; $55 \mathrm{mg} / 100 \mathrm{~mL}$ protein; glucose not done) in the CSF. The infant died suddenly at 8 days of age.

Autopsy revealed bile-stained ascites, an enlarged liver (142 g, normal $80 \mathrm{~g}$ ), retroperitoneal and pancreatic edema, and an enlarged heart (26 g, normal $18 \mathrm{~g}$ ). Microscopy revealed diffuse myocarditis involving both atria and ventricles, which was apparent on microscopy. Foci of early myocardial calcification were present in the wall of the right atrium. Inflammation (lymphocytes and microglia) of the spinal leptomeninges and spinal cord was present at the cervical, thoracic, and lumbar levels. The remainder of the brain was unremarkable; specifically there was no ventriculitis or meningitis. Coxsackie B4 virus was cultured from the CSF, pancreas, intestines, spleen, and lung, but not from the brain. The cause of death was attributed to neonatal myocarditis and meningomyelitis due to Coxsackie B4 virus, possibly acquired in utero or at the time of birth given the maternal symptoms prior to delivery.

\section{Summary of Other Cases}

In addition to the three cases presented in detail, two male infants who died at 2 and 3 days of age from congenital heart defect had elevation of antibody titers to Coxsackie B2 and B4 (one each). Neither had evidence of heart or brain inflammation and virus was not isolated. A 2.5-year-old male died of Coxsackie A9 bronchopneumonia; however, the brain and heart were normal. A 5-year-old male presented with seizures and died of septic shock. Myocarditis, pancreatitis, orchitis, and bronchopneumonia suggested a viral etiology, with Coxsackie as a likely candidate, but cultures were unsuccessful.

\section{Discussion}

The fetus and neonate are particularly susceptible to Coxsackie B virus infections, particularly of the CNS. ${ }^{20,21}$ Common manifestations are meningitis and/or encephalitis, as 
evidenced in Cases 1 and 3; however, anatomic defects of the brain and abnormal neurodevelopment have also been reported. ${ }^{16,18}$

Traditionally, wider viral dissemination is attributed to the limited immune response of the fetus/neonate. ${ }^{15,21}$ This explanation is likely insufficient. Based on a study of neonatal mice injected intracerebrally with Coxsackie B3 virus, Feuer et al showed that the virus targets neonatal neuronal stem cells, particularly around the cerebral ventricles. ${ }^{20}$ Virus is then carried into the brain parenchyma by developing neurons, which continue to migrate and differentiate despite infection. In the neonatal mice, the susceptibility to infection decreased dramatically within days of birth. Upon maturation, the infected neurons undergo selective apoptosis. The authors postulate that this neuronal loss may correlate with the neurologic sequelae associated with Coxsackie viral infections. ${ }^{20}$ This might explain the hemorrhagic and necrotic features described on neuropathology in Case 2.

The age-dependent susceptibility to infection likely also relates to changes in expression of viral receptors. Coxsackieviruses and adenoviruses share a common receptor, the coxsackievirus and adenovirus receptor (CAR). ${ }^{22}$ The CAR protein is expressed during fetal development, particularly in the CNS and muscle but also in the liver, lung, heart, and pancreas. $^{22}$ It is purported that CAR also functions as a mediator of cell adhesion involved in neural-network formation in the developing brain. ${ }^{23}$ In mice, a rapid downregulation of CAR expression occurs at birth. ${ }^{22}$ This likely explains why Case 1, a 22-week fetus, had severe ventriculitis whereas the CNS changes in Case 3, a term infant, were milder.

Ventriculitis associated with Coxsackie virus infection is very rare. There is only one case reported in which a 7-weekold male infant was diagnosed with Coxsackie B1 meningitis and ventriculitis by lumbar puncture and magnetic resonance imaging. ${ }^{13}$ The child survived. Our case of fetal ventriculitis is unique for several reasons. First, Coxsackie B4 virus was isolated. Second, ventriculomegaly was appreciated antenatally on ultrasound. Finally, the resultant severe ventriculitis was the predominant neurological abnormality on autopsy.

Brain involvement with Coxsackie B viral infections has been previously described. A 30-week fetus with Coxsackie B3 infection had total liquefactive necrosis of the brain along with meningoencephalitis, interstitial pneumonitis, mild myocardial hypertrophy, and chronic monocytic placental villitis. ${ }^{9}$ The findings in our Case 3 with predominantly spinal cord involvement are similar to a previously reported case. ${ }^{24}$ Another autopsy report describes myocarditis and foci of necrosis and inflammation in the brain of a 6-day-old female with Coxsackie B2 infection, as well as pneumonitis and widespread perivascular inflammation in the spinal cord and brain of a 21-month-old male with Coxsackie B4 infection. ${ }^{25}$ A 4-year-old male with Coxsackie B1 pneumonitis had meningoencephalitis affecting the temporal lobes, basal nuclei, and brainstem. ${ }^{19}$ A 33-year-old female died with meningoencephalitis and midbrain damage caused by Coxsackie B4 infection. $^{26}$

Our case of fetal hydrops secondary to Coxsackie B4 myocarditis is also distinctive, as there have been few reports with an antenatal diagnosis. ${ }^{12,17}$ Ouellet et al described a case of hydrops fetalis in which Coxsackie B3 was isolated from the amniotic fluid. ${ }^{12}$ In contrast to our case, mild cardiac involvement and no neurologic abnormalities were found on autopsy. All three perinatal cases in our series reflect the propensity for myocardial involvement.

Enteroviral infection may be detected by serology, viral culture, or nucleic acid amplification. ${ }^{4}$ Many enteroviruses require 4 to 10 days to grow in tissue culture ${ }^{6,7}$; therefore, results may not be available to direct early patient management and viral culture is further limited by poor sensitivity $(<75 \%)^{4,19}$ This may account for the lack of growth from postmortem tissues in Case 1.

Serological testing for enteroviral infection is not clinically useful. ${ }^{3}$ It is time consuming owing to the large number of serotypes. ${ }^{4}$ In our center, Coxsackie virus serology is no longer available. Nucleic acid amplification by PCR is currently the preferred method for diagnosis due to a rapid turnaround of results ( $<24$ hours) and high sensitivity. 3,4,6

In utero diagnosis of a Coxsackie $B$ viral infection is challenging because there is no access to traditional sample sources such as respiratory secretions, blood, CSF, stool, and urine. Transplacental infection can be inferred from the isolation of virus in the amniotic fluid, as in Case 1. Other reports have also relied on this method of diagnosis. ${ }^{12,17,27}$

Placental pathology may reveal evidence of an intrauterine infection. Inflammation of the placental villi is characteristic of infection reaching the placenta from the maternal bloodstream. ${ }^{9}$ This has been shown in fatal cases of fetal Coxsackie B3 and A9 infections. ${ }^{9,28}$ Euscher et al demonstrated Coxsackie virus by PCR in placentas of neonates with neurodevelopmental delay. ${ }^{16}$ In addition to chronic villitis, focal calcification and hemorrhagic vasculitis were noted on placental examination. ${ }^{16}$ Diffuse perivillous fibrin deposition and villous necrosis have also been described. ${ }^{28}$ Unfortunately the placentas of our two neonatal cases had not been examined.

In our present fetal case, transplacental infection was shown by the viral isolation from the amniotic fluid and by the finding of chronic villitis with dystrophic calcifications. We were unable to detect viral inclusions by electron microscopy of the placenta. Kamei et al reported a similar difficulty and suggested that the relatively small size of the Coxsackie virus (25 to $30 \mathrm{~nm}$ ) compared with other viruses may be responsible for the negative findings. ${ }^{19}$

The pregnancy in Case 1 was terminated following of the development of suspected maternal mirror syndrome, a recognized complication of hydrops. Furthermore, termination was recommended because the fetal prognosis was poor. The extent of disease in the first case was similar to the presentation in Cases 2 and 3, both with unfavorable outcomes. Mortality associated with neonatal Coxsackie B4 infection is as high as $40 \%$, which is greater than other group B serotypes. ${ }^{3}$ Acquisition of infection, either in utero or perinatally, is the most significant risk factor leading to fatal disease. $^{2}$

No vaccines exist for the primary prevention of Coxsackie $B$ disease. ${ }^{3}$ Intravenous immunoglobulin has been used in the 
treatment of neonatal enteroviral myocarditis; however, there is no clear evidence for its effectiveness. ${ }^{6,10}$ Pleconaril, an antipicornaviral agent, binds to the viral capsid and prevents viral uncoating within the host cell. ${ }^{4}$ Pleconaril has antiviral activity against more than $90 \%$ of the commonly circulating enterovirus serotypes. ${ }^{11}$ Reports of its use in neonatal populations with Coxsackie B infections have shown conflicting results. ${ }^{6,10,11}$ Although pleconaril prevents further viral replication, it does not reverse tissue damage that has already occurred. Thus, the drug may have little effect in infants with established multiorgan disease and tissue damage. $^{6,7}$ Currently, pleconaril is no longer available for use. ${ }^{10}$

\section{Acknowledgments}

Consent for the publication of case details was obtained from the patient in Case 1. As Case 2 and Case 3 were remote, the authors were unable to contact the parents involved for consent. Dr. Del Bigio holds the Canada Research Chair in Developmental Neuropathology.

\section{References}

1 Moore M, Kaplan MH, McPhee J, Bregman DJ, Klein SW. Epidemiologic, clinical, and laboratory features of Coxsackie B1-B5 infections in the United States, 1970-79. Public Health Rep 1984;99 (5):515-522

2 Kaplan MH, Klein SW, McPhee J, Harper RG. Group B coxsackievirus infections in infants younger than three months of age: a serious childhood illness. Rev Infect Dis 1983;5(6):1019-1032

3 Romero JR. Pediatric group B coxsackievirus infections. Curr Top Microbiol Immunol 2008;323(3):223-239

4 Sawyer MH. Enterovirus infections: diagnosis and treatment. Curr Opin Pediatr 2001;13(1):65-69

5 Theodoridou M, Kakourou T, Laina I, Mostrou G, Tsakris A. Vesiculopapular rash as a single presentation in intrauterine coxsackie virus infection. Eur J Pediatr 2002;161(7):412-413

6 Bryant PA, Tingay D, Dargaville PA, Starr M, Curtis N. Neonatal coxsackie B virus infection-a treatable disease? Eur J Pediatr 2004;163(4-5):223-228

7 Callen J, Paes BA. A case report of a premature infant with coxsackie B1 meningitis. Adv Neonatal Care 2007;7(5):238-247

8 Cheng LL, Ng PC, Chan PK, Wong HL, Cheng FW, Tang JW. Probable intrafamilial transmission of coxsackievirus b3 with vertical transmission, severe early-onset neonatal hepatitis, and prolonged viral RNA shedding. Pediatrics 2006;118(3):e929-e933

9 Konstantinidou A, Anninos H, Spanakis N, et al. Transplacental infection of Coxsackievirus B3 pathological findings in the fetus. J Med Virol 2007;79(6):754-757

10 Freund MW, Kleinveld G, Krediet TG, van Loon AM, VerboonMaciolek MA. Prognosis for neonates with enterovirus myocarditis. Arch Dis Child Fetal Neonatal Ed 2010;95(3):F206-F212
11 Bauer S, Gottesman G, Sirota L, Litmanovitz I, Ashkenazi S, Levi I. Severe Coxsackie virus B infection in preterm newborns treated with pleconaril. Eur J Pediatr 2002;161(9):491-493

12 Ouellet A, Sherlock R, Toye B, Fung KF. Antenatal diagnosis of intrauterine infection with coxsackievirus B3 associated with live birth. Infect Dis Obstet Gynecol 2004;12(1):23-26

13 Erickson LS, Hoyle G, Abramson J, Hester LC, Shetty AK. Coxsackie B1 infection associated with ventriculitis. Pediatr Infect Dis J 2003;22(8):750-751

14 Dahlquist G, Frisk G, Ivarsson SA, Svanberg L, Forsgren M, Diderholm $\mathrm{H}$. Indications that maternal coxsackie B virus infection during pregnancy is a risk factor for childhood-onset IDDM. Diabetologia 1995;38(11):1371-1373

15 Tracy S, Gauntt C. Group B coxsackievirus virulence. Curr Top Microbiol Immunol 2008;323;49-63

16 Euscher E, Davis J, Holzman I, Nuovo GJ. Coxsackie virus infection of the placenta associated with neurodevelopmental delays in the newborn. Obstet Gynecol 2001;98(6):1019-1026

17 Watson WJ, Awadallah S, Jaqua MJ. Intrauterine infection with coxsackievirus: is it a cause of congenital cardiac malformations? Infect Dis Obstet Gynecol 1995;3(2):79-81

18 Gauntt CJ, Gudvangen RJ, Brans YW, Marlin AE. Coxsackievirus group $B$ antibodies in the ventricular fluid of infants with severe anatomic defects in the central nervous system. Pediatrics 1985;76(1):64-68

19 Kamei S, Hersch SM, Kurata T, Takei Y. Coxsackie B antigen in the central nervous system of a patient with fatal acute encephalitis: immunohistochemical studies of formalin-fixed paraffin-embedded tissue. Acta Neuropathol 1990;80(2):216-221

20 Feuer R, Mena I, Pagarigan RR, Harkins S, Hassett DE, Whitton JL. Coxsackievirus B3 and the neonatal CNS: the roles of stem cells, developing neurons, and apoptosis in infection, viral dissemination, and disease. Am J Pathol 2003;163(4):1379-1393

21 Feuer R, Whitton JL. Preferential coxsackievirus replication in proliferating/activated cells: implications for virus tropism, persistence, and pathogenesis. Curr Top Microbiol Immunol 2008; 323;149-173

22 Freimuth P, Philipson L, Carson SD. The coxsackievirus and adenovirus receptor. Curr Top Microbiol Immunol 2008;323; 67-87

23 Honda T, Saitoh H, Masuko M, et al. The coxsackievirus-adenovirus receptor protein as a cell adhesion molecule in the developing mouse brain. Brain Res Mol Brain Res 2000;77(1):19-28

24 Benirschke K, Kibrick S. Acute aseptic myocarditis and meningoencephalitis in the newborn child infected with coxsackle virus group B, type 3. N Engl J Med 1956;255(19):883-889

25 Estes ML, Rorke LB. Liquefactive necrosis in Coxsackie B encephalitis. Arch Pathol Lab Med 1986;110(11):1090-1092

26 Cree BC, Bernardini GL, Hays AP, Lowe G. A fatal case of coxsackievirus B4 meningoencephalitis. Arch Neurol 2003;60(1): 107-112

27 Strong BS, Young SA. Intrauterine coxsackie virus, group B type 1, infection: viral cultivation from amniotic fluid in the third trimester. Am J Perinatol 1995;12(2):78-79

28 Batcup G, Holt P, Hambling MH, Gerlis LM, Glass MR. Placental and fetal pathology in Coxsackie virus A9 infection: a case report. Histopathology 1985;9(11):1227-1235 